\title{
PRODUTIVIDADE DA CULTIVAR DE UVA DE MESA NIAGARA ROSADA SOBRE DIFE- RENTES PORTA-ENXERTOS, EM MONTE ALEGRE DO SUL-SP ${ }^{1}$
}

\author{
MAURILO MONTEIRO TERRA ${ }^{2,3}$, ERASMO JOSÉ PAIOLI PIRES ${ }^{2,3}$, CELSO VALDEVINO POMMER $^{2,3}$, \\ RENATO VASCONCELOS BOTELHO ${ }^{2,4}$
}

\begin{abstract}
RESUMO - Com o objetivo de estudar o comportamento da cultivar de uva de mesa Niagara Rosada enxertada sobre diferentes porta-enxertos, um experimento foi conduzido na região de Monte Alegre do Sul-SP, durante seis anos consecutivos. Os porta-enxertos estudados foram 'Schwarzmann', IAC 572 'Jales', IAC 313 'Tropical', 'Traviú', IAC 766 'Campinas' e 'Kober 5BB'. Os porta-enxertos IAC-313 'Tropical', IAC 766 'Campinas' e 'Traviú' são recomendados para a uva de mesa 'Niagara Rosada' na região de Monte Alegre do Sul-SP. A cultivar Niagara Rosada sobre o porta-enxerto 'Kober 5BB' teve menor produtividade em comparação com os demais porta-enxertos avaliados, não sendo recomendado para a região de Monte Alegre do Sul-SP.
\end{abstract}

Termos para indexação: videira, Vitis, uva, produtividade, porta-enxertos.

\section{YIELD OF THE NIAGARA ROSADA TABLE GRAPE CULTIVAR GRAFTED ON DIFFERENT ROOTSTOCKS, IN MONTE ALEGRE DO SUL-SP}

\begin{abstract}
With the objective of studying the behavior of the Niagara Rosada table grape cultivar grafted on different rootstocks, a trial was carried out in the region of Monte Alegre do Sul-SP, Brazil, by six consecutive years. The rootstocks studied were 'Schwarzmann', IAC 572 'Jales', IAC 313 'Tropical', 'Traviú', IAC 766 'Campinas' e 'Kober 5BB'. It was verified from the results obtained, that the rootstocks IAC 313 'Tropical', IAC 766 'Campinas' and 'Traviú' are recommend for the 'Niagara Rosada' for the region of Monte Alegre do Sul-SP. The Niagara Rosada grafted on the rootstock 'Kober 5BB' had a minor yield per vine compared with the others rootstocks, and can not be recommended for the region of Monte Alegre do Sul-SP.
\end{abstract}

Index terms: grapevine, Vitis, yield, grape, rootstocks.

A uva 'Niagara Rosada' é o resultado de uma mutação somática ocorrida na uva 'Niagara Branca' (Vitis labrusca L. x Vitis vinifera L.) em 1933, em Louveira-SP, que rapidamente predominou sobre a forma original (Sousa, 1996). Na viticultura paulista as Niagaras são as uvas mais cultivadas, perfazendo uma área de 7.626 hectares, o que representa $66,3 \%$ dos vinhedos (Ghilardi \& Maia, 2001).

Com a disseminação da filoxera, um afídio que danifica as raízes da videira, os viticultores tiveram que fazer uso de porta-enxertos tolerantes ou resistentes a esta praga. Entretanto, apesar da disponibilidade razoável de bons porta-enxertos, cada um deles tem a sua limitação, e só a experimentação regional poderá determinar qual é o mais adequado para cada condição de cultivo (Pommer et al., 1997).

Em estudo desenvolvido na região de Jundiaí-SP com a cultivar Niagara Rosada, as melhores produções foram obtidas quando utilizou-se o porta-enxerto 'Schwarzmann', o qual não diferiu dos portaenxertos 'Golia', 'Traviú', 'IAC 572' e 'IAC 571-6'. No entanto, os portaenxertos não influenciaram o peso médio dos cachos (Terra et al., 1988).

Na região do Vale do Paraíba-SP, Pauletto (1999), comparando diferentes porta-enxertos para a cultivar Niagara Rosada, verificou maiores produções quando as plantas foram enxertadas sobre 'IAC 766', 'IAC 313' e 'Traviú', superando os porta-enxertos 'Kober 5BB' e 'Schwarzmann'.

Em outro experimento conduzido na região de Mococa-SP por cinco anos consecutivos, as melhores produções para a uva 'Niagara Rosada' foram observadas quando utilizaram-se os porta-enxertos 'IAC 572' e 'IAC 313' e maior desenvolvimento vegetativo com os portaenxertos 'IAC 766' ' 'IAC 313' (Terra et al., 2002). Para a cultivar Concord, também na região de Mococa-SP, Terra et al. (2001) verificaram maiores produções quando as plantas foram enxertadas sobre 'IAC 313', 'IAC 571-6' ' 'IAC 572'. A pior performance foi constatada quando se utilizou o porta-enxerto 'IAC 766'.

No município de Caldas-MG, Alvarenga et al. (2002) observaram que o porta-enxerto 'IAC 766' induziu a produção do maior número de cachos para a cultivar Niagara Rosada. Para a variável produção total, estes autores verificaram superioridade quando as plantas foram enxertadas sobre os porta-enxertos 'IAC 766' e 'IAC 572'.

Para a região de Jales-SP e outras localidades de clima tropical, Maia \& Camargo (2002) recomendam o uso do porta-enxerto 'IAC 572' para o cultivo da 'Niagara Rosada', no sistema de latada. Por outro lado, para as regiões de Jundiaí e São Miguel Arcanjo-SP, Braga (1988) sugere a utilização dos porta-enxertos 'IAC 766' e 'Traviú'.

Em Monte Alegre do Sul-SP, em trabalho onde se estudou o comportamento da cultivar de uva para vinho IAC 138-22 'Máximo' sobre diferentes porta-enxertos, Terra et al. (1990) verificaram maiores produções quando as plantas foram enxertadas sobre 'IAC-572'.

Tendo em vista a grande variação de comportamento da combinação copa x porta-enxerto para diferentes condições edafo-climáticas, este trabalho teve como objetivo avaliar a produtividade da cultivar de uva de mesa Niagara Rosada sobre diferentes porta-enxertos, na região de Monte Alegre do Sul-SP.

O experimento foi instalado em 1993 na Estação Experimental de Monte Alegre do Sul-SP, do Instituto Agronômico de Campinas, situada a latitude de $22^{\circ} 43^{\prime} \mathrm{S}$ e longitude $46^{\circ} 37^{\prime} \mathrm{O}, 740 \mathrm{~m}$ de altitude, clima classificado como Cwa e, em solo argissolo vermelho-amarelo.

A cultivar utilizada como copa foi a Niagara Rosada, plantada no espaçamento $2 \times 1 \mathrm{~m}$ e conduzida no sistema de espaldeira com três fios de arame e poda curta de inverno, com uma gema, no cordão esporonado unilateral.

Os porta-enxertos estudados foram 'Schwarzmann', IAC 572 'Jales', IAC 313 'Tropical', 'Traviú', IAC 766 'Campinas' e 'Kober 5BB', cujas características são apresentadas a seguir:

Schwarzmann - Híbrido natural entre Vitis riparia e Vitis rupestris, selecionado na Moravia, por Bizenz. Apresenta vigor médio, adaptado a terrenos secos, áridos, ácidos e arenosos, com bom pegamento (Pommer et al., 1997).

IAC 572 'Jales' - Obtido do cruzamento entre Vitis caribaea e 101-14 Mgt, efetuado por Santos Neto e lançado ao cultivo em 1970. Apresenta alto vigor, adaptado tanto a solos argilosos como arenosos,

\footnotetext{
${ }^{1}$ (Trabalho 142/2002). Recebido: 10/09/2002. Aceito para publicação: 11/09/2003.

${ }^{2}$ Eng. Agr. Dr. Instituto Agronômico (IAC). Caixa Postal 28, CEP: 13001-970, Campinas-SP. E-eletrônico: mmterra@iac.br.

${ }^{3}$ Bolsista CNPq.

${ }^{4}$ Bolsista FAPESP.
} 
TABELA 1 - Produção por planta $(\mathrm{kg})$ da cultivar de uva de mesa Niagara Rosada sobre diferentes porta-enxertos, em seis anos agrícolas consecutivos, em Monte Alegre do Sul-SP.

\begin{tabular}{|c|c|c|c|c|c|c|c|}
\hline \multirow[t]{2}{*}{ Porta -enxerto } & \multicolumn{6}{|c|}{ Anos de Produção } & \multirow[t]{2}{*}{ Média } \\
\hline & 1996 & 1997 & 1998 & 1999 & 2000 & 2001 & \\
\hline Schwarzmann & 2,16 bc $\quad \mathrm{C}$ & $2,72 \mathrm{a} \quad \mathrm{BC}$ & $3,44 \mathrm{a} A$ & $3,17 \mathrm{ab} \mathrm{AB}$ & 3,09 bc $\mathrm{AB}$ & $2,66 \mathrm{a} \quad \mathrm{BC}$ & $2,88 \mathrm{~b}$ \\
\hline IAC 572 & 2,85 b A & $2,54 \mathrm{a} A$ & $2,99 \mathrm{a} A$ & 2,88 b A & 2,88 с A & $2,70 \mathrm{a} A$ & $2,81 \mathrm{~b}$ \\
\hline IAC 313 & $3,59 \mathrm{a} \quad \mathrm{AB}$ & $2,40 \mathrm{a}$ & $3,37 \mathrm{a} \quad \mathrm{AB}$ & $3,82 \mathrm{a} \quad \mathrm{A}$ & $3,82 \mathrm{a} \quad \mathrm{A}$ & $2,97 \mathrm{a} \quad \mathrm{BC}$ & $3,33 \mathrm{a}$ \\
\hline Traviú & $2,74 \mathrm{~b} \quad \mathrm{~B}$ & $2,97 \mathrm{a} \quad \mathrm{AB}$ & $3,39 \mathrm{a} \quad \mathrm{AB}$ & 3,53ab A & $3,54 a b c A$ & $3,04 \mathrm{a} A B$ & $3,20 \mathrm{a}$ \\
\hline IAC 766 & 2,32 bc $\quad C$ & $2,68 \mathrm{a} \quad \mathrm{BC}$ & $3,66 \mathrm{a} A$ & $3,59 \mathrm{a} \quad \mathrm{A}$ & $3,67 \mathrm{ab} \quad \mathrm{A}$ & $3,20 \mathrm{a} \quad \mathrm{AB}$ & $3,19 \mathrm{a}$ \\
\hline Kober 5BB & $1,76 \mathrm{cA}$ & $1,64 \mathrm{bA}$ & $1,80 \mathrm{~b} \mathrm{~A}$ & $1,73 \mathrm{cA}$ & $1,62 \mathrm{dA}$ & $1,50 \mathrm{bA}$ & $1,68 \mathrm{c}$ \\
\hline CV $(\%)$ & 9,96 & & & & & & \\
\hline Fatores & Ano & Por & erto & Ano & ta-enxerto & & \\
\hline $\mathrm{F}$ & $5,69 * *$ & & & & p** & & \\
\hline
\end{tabular}

Médias seguidas pela mesma letra minúscula (coluna) e mesma letra maiúscula (linha), não diferem entre si pelo teste de Tukey a 1\% de probabilidade.

folhas resistentes às principais doenças, ótimo enraizamento e pegamento (Pommer et al., 1997).

IAC 313 ‘ Tropical' - Originário do cruzamento do porta-enxerto 'Golia' com a espécie de videira tropical Vitis cinerea realizado por Santos Neto. Apresenta alto vigor, adaptando-se bem a diferentes tipos de solos e suas folhas têm boa resistência às moléstias (Pommer et al., 1997).

Traviú - Híbrido entre Vitis riparia $\mathrm{x}$ (Vitis rupestris x Vitis cordifolia), obtido por Millardet e De Grasset, na França, em 1882, introduzido como Vitis riparia em Jundiaí. Possui bom desenvolvimento, porém pouco vigoroso, mas com boa adaptação a solos ácidos (Pommer et al., 1997).

IAC 766 'Campinas' - Obtido pelo cruzamento do porta-enxerto Traviú com a espécie de videira tropical Vitis caribaea realizado por Santos Neto, em 1958. Apresenta alto vigor e boa adaptação às condições edafo-climáticas paulistas; suas folhas são bastante resistentes às doenças (Pommer et al., 1997).

Kober 5BB - Resultado do cruzamento entre as espécies Vitis berlandierie Vitis riparia, realizado por Teleki e selecionado por Kober, na Áustria, no início do século XX. Apresenta vigor médio e boa adaptação a diferentes tipos de solo e boa resistência à seca e às doenças fúngicas (Pommer et al., 1997).

O delineamento experimental foi em blocos casualizados, com seis tratamentos e quatro repetições, totalizando 24 parcelas, cada qual constituída de 12 plantas.

Durante seis anos consecutivos, de 1996 a 2001, foram avaliadas as variáveis produção total por planta, expressa em quilogramas; número de cachos por planta e peso médio dos cachos, em gramas.

Os resultados foram submetidos à análise de variância conjunta, com agrupamento por ano agrícola e comparação de médias através do teste de Tukey a $1 \%$ de probabilidade.

No primeiro ano de avaliação, 1996, a maior produtividade foi verificada para as plantas enxertadas sobre o porta-enxerto 'IAC 313', diferindo significativamente dos demais porta-enxertos. Nos outros anos, este porta-enxerto também conferiu maior produtividade às plantas, po- rém nos $2^{\circ}, 3^{\circ}$ e $6^{\circ}$ anos foi superior somente ao porta-enxerto 'Kober $5 B B^{\prime}$. No $4^{\circ}$ ano de avaliação foi também melhor que o porta-enxerto 'IAC 572' e, no $5^{\circ}$ ano superou ainda o 'Schwarzmann'. Pela comparação entre as médias dos seis anos, os porta-enxertos 'IAC 313', 'Traviú' e 'IAC 766' induziram as maiores produções por parcela sem diferirem estatisticamente entre si (Tabela 1).

Os porta-enxertos 'IAC 572' e 'Kober 5BB' foram os que apresentaram menor alternância de produção, sendo que não houve diferenças significativas entre os diferentes anos de avaliação (Tabela 1).

Em todos os anos, o porta-enxerto 'Kober 5BB' induziu a produção de um menor número de cachos por planta, diferindo significativamente de pelo menos um dos outros porta-enxertos estudados. Os demais porta-enxertos não diferiram estatisticamente entre si. Na média dos seis anos de estudo, todos os porta-enxertos conferiram um número de cachos por planta superior ao 'Kober 5BB'. A maior variação de número de cachos produzidos entre os anos de estudo foi observado para o porta-enxerto 'IAC-313' (Tabela 2).

No primeiro ano do experimento, os porta-enxertos 'IAC-572', 'IAC-313' e 'Traviú' induziram maior peso médio dos cachos. No entanto, nos outros anos, somente as plantas enxertadas sobre 'Kober 5BB' apresentaram peso médio de cachos significativamente inferior aos demais. Variações entre os diferentes anos somente foram verificadas para os porta-enxertos 'IAC-572' e 'IAC-766' (Tabela 3).

Os resultados deste experimento são parcialmente condizentes com aqueles obtidos por Terra et al. (2002) que verificaram maior produção da uva 'Niagara Rosada' em Mococa-SP, com os porta-enxertos 'IAC-572' e 'IAC 313' e menor produção com o 'IAC-766'. De forma semelhante, Terra et al. (1988), em experimento realizado em Jundiaí-SP, verificaram bom desempenho não somente do porta-enxerto 'IAC-572', como também do 'Traviú', do 'Schwarzmann' e do 'IAC-571-6'.

Por outro lado, no presente trabalho o porta enxerto 'IAC-572' induziu menor produtividade, enquanto que o 'IAC-766' possibilitou produtividade superior. Estas divergências de resultados evidenciam a importância da realização de experimentos regionais para a melhor indicação da combinação copa/porta-enxerto, tendo em vista as diferenças

TABELA 2 - Número de cachos por planta da cultivar de uva de mesa Niagara Rosada sobre diferentes porta-enxertos, em seis anos agrícolas consecutivos, em Monte Alegre do Sul-SP.

\begin{tabular}{|c|c|c|c|c|c|c|c|}
\hline \multirow[t]{2}{*}{ Porta -enxerto } & \multicolumn{6}{|c|}{ Anos de Produção } & \multirow[t]{2}{*}{ Média } \\
\hline & 1996 & 1997 & 1998 & 1999 & 2000 & 2001 & \\
\hline Schwarzmann & 11,18 b B & $12,04 \mathrm{ab} \mathrm{B}$ & $15,58 \mathrm{a} A$ & $14,36 \mathrm{a} \quad \mathrm{AB}$ & $14,03 \mathrm{a} A B$ & $12,61 \mathrm{a} A B$ & $13,30 \mathrm{a}$ \\
\hline IAC 572 & $10,99 \mathrm{bA}$ & $12,10 \mathrm{abA}$ & $12,98 \mathrm{a} \mathrm{A}$ & $12,77 \mathrm{abA}$ & $12,92 \mathrm{abA}$ & $12,35 \mathrm{a} \mathrm{A}$ & $12,35 \mathrm{a}$ \\
\hline IAC 313 & $15,21 \mathrm{a} A B$ & $11,02 \mathrm{ab} \quad \mathrm{C}$ & $14,23 \mathrm{a} \quad \mathrm{ABC}$ & $15,91 \mathrm{a} A$ & $15,58 \mathrm{a} A$ & $12,21 \mathrm{ab} \mathrm{BC}$ & $14,03 \mathrm{a}$ \\
\hline Traviú & $12,25 \mathrm{abA}$ & $13,32 \mathrm{aA}$ & $14,52 \mathrm{a} \mathrm{A}$ & $14,66 \mathrm{a} A$ & $14,81 \mathrm{a} \mathrm{A}$ & $12,45 \mathrm{a} \quad \mathrm{A}$ & $13,67 \mathrm{a}$ \\
\hline IAC 766 & $11,30 \mathrm{~b} \mathrm{~B}$ & $12,49 \mathrm{abAB}$ & $15,05 \mathrm{a} A$ & $14,99 \mathrm{a} A$ & $15,21 \mathrm{aA}$ & $13,62 \mathrm{a} A B$ & $13,78 \mathrm{a}$ \\
\hline Kober 5BB & $10,21 \mathrm{bA}$ & $9,36 \mathrm{bA}$ & $9,74 \mathrm{bA}$ & $10,22 \mathrm{bA}$ & $9,83 \mathrm{bA}$ & $9,08 \mathrm{bA}$ & $9,74 \mathrm{~b}$ \\
\hline CV (\%) & 10,34 & & & & & & \\
\hline Fatores & Ano & \multirow{2}{*}{\multicolumn{2}{|c|}{$\begin{array}{c}\text { Porta-enxerto } \\
15,77^{* *}\end{array}$}} & \multicolumn{2}{|c|}{ Ano x Porta-enxerto } & & \\
\hline $\mathrm{F}$ & $6,39 * *$ & & & & $26 * *$ & & \\
\hline
\end{tabular}

Médias seguidas pela mesma letra minúscula (coluna) e mesma letra maiúscula (linha), não diferem entre si pelo teste de Tukey a 1\% de probabilidade. 
TABELA 3 - Peso médio (g) dos cachos da cultivar de uva de mesa Niagara Rosada sobre diferentes porta-enxertos, em seis anos agrícolas consecutivos, em Monte Alegre do Sul-SP.

\begin{tabular}{|c|c|c|c|c|c|c|c|}
\hline \multirow[t]{2}{*}{ Porta -enxerto } & \multicolumn{6}{|c|}{ Anos de Produção } & \multirow[t]{2}{*}{ Média } \\
\hline & 1996 & 1997 & 1998 & 1999 & 2000 & 2001 & \\
\hline Schwarzmann & 204,71 bc A & $227,49 \mathrm{a} \mathrm{A}$ & $221,08 \mathrm{a} \mathrm{A}$ & $220,51 \mathrm{a} \mathrm{A}$ & $220,19 \mathrm{a} \mathrm{A}$ & $213,18 \mathrm{a} \mathrm{A}$ & $217,86 \mathrm{a}$ \\
\hline IAC 572 & $258,72 \mathrm{a} \quad \mathrm{A}$ & $212,50 a \quad B$ & $231,6 \mathrm{Aa} \quad \mathrm{B}$ & $225,92 \mathrm{a} A B$ & $223,80 a \quad B$ & $218,94 a \quad B$ & $228,58 \mathrm{a}$ \\
\hline IAC 313 & $236,39 \mathrm{ab} \quad \mathrm{A}$ & $219,12 \mathrm{a} \mathrm{A}$ & $237,37 \mathrm{a} \mathrm{A}$ & $240,31 \mathrm{a} \mathrm{A}$ & $245,71 \mathrm{a} \mathrm{A}$ & $242,94 a \mathrm{~A}$ & $236,98 \mathrm{a}$ \\
\hline Traviú & $224,46 \mathrm{ab} \quad \mathrm{A}$ & $224,77 \mathrm{a} A$ & $233,72 \mathrm{a} A$ & $241,04 \mathrm{a} A$ & $239,13 \mathrm{a} A$ & $246,11 \mathrm{aA}$ & $234,87 \mathrm{a}$ \\
\hline IAC 766 & 204,93 bc B & $214,55 \mathrm{a} A B$ & $242,82 \mathrm{aA}$ & $239,16 \mathrm{a} A B$ & $240,80 \mathrm{a} A$ & $234,77 \mathrm{a} A B$ & $229,50 \mathrm{a}$ \\
\hline Kober 5BB & $171,72 \quad \mathrm{dA}$ & $177,51 \mathrm{bA}$ & $185,84 \mathrm{bA}$ & $169,60 \mathrm{bA}$ & $165,08 \mathrm{bA}$ & $165,32 \mathrm{bA}$ & $172,5 \quad b$ \\
\hline CV $(\%)$ & 6,42 & & & & & & \\
\hline Fatores & Ano & \multicolumn{2}{|c|}{ Porta-enxerto } & \multicolumn{2}{|c|}{ Ano x Porta-enxerto } & & \\
\hline $\mathrm{F}$ & $0,94^{\text {n.s. }}$ & \multicolumn{2}{|c|}{$26,07 * *$} & \multicolumn{2}{|c|}{$2,70 * *$} & & \\
\hline
\end{tabular}

Médias seguidas pela mesma letra minúscula (coluna) e mesma letra maiúscula (linha), não diferem entre si pelo teste de Tukey a $1 \%$ de probabilidade.

edafo-climáticas de cada região.

O peso médio dos cachos, o número de cachos e a produção por planta da uva 'Niagara Rosada' foram drasticamente reduzidos em plantas enxertadas sobre 'Kober 5BB', possivelmente devido ao seu baixo vigor e pouca adaptabilidade aos solos ácidos (Pommer et al., 1997), não podendo, portanto, ser recomendado para o cultivo desta variedade.

Os porta-enxertos IAC-313 'Tropical', IAC 766 'Campinas' e 'Traviú' são recomendados para a uva de mesa 'Niagara Rosada', na região de Monte Alegre do Sul-SP.

A cultivar Niagara Rosada sobre o porta-enxerto 'Kober 5BB' teve menor produtividade em comparação com os demais porta-enxertos avaliados, não sendo recomendado para a região de Monte Alegre do Sul-SP.

\section{REFERÊNCIAS BIBLIOGRÁFICAS}

ALVARENGA, A. A.; REGINA, M. A.; FRÁGUAS, J. C.; SILVA, A. L.; SOUZA, C. M.; CANÇADO, G. M.A.; FREITAS, G F. Indicação de porta-enxertos de videiras para o sul de Minas Gerais. In: REGINA, M.A. (Ed.) Viticultura e enologia: atualizando conceitos. Caldas: Epamig-FECD, 2002.p. 243-256.

BRAGA, F. G. Cultura da uva Niágara Rosada. São Paulo: Nobel, 1988. $66 \mathrm{p}$.

GHILARDI, A. A.; MAIA, M. L. Tecnologia, custo de produção e rentabilidade do cultivo de uva Niagara no Estado de São Paulo. Informações Econômicas, São Paulo, v.31, n.12, p. 48-64, 2001.

MAIA, J. D. G.; CAMARGO, U.A. Implantação do vinhedo e manejo das plantas. In: MAIA, J. D. G.; KUHN, G. B. (Ed.)Cultivo da Niagara
Rosada em regiões tropicais do Brasil. Bento Gonçalves: EmbrapaSPI; Embrapa-CNPUV, 2002.p. 13-23.

PAULETTO, D. Avaliação de porta-enxertos para a videira 'Niagara Rosada` no Vale do Paraíba, SP. 51 f. Dissertação (Mestrado em Fitotecnia) Escola Superior de Agricultura “Luiz de Queiroz”, Universidade de São Paulo, Piracicaba, 1999.

POMMER, C. V.; PASSOS, I. R. S.; TERRA, M. M.; PIRES, E. J. P. Variedades de videira para o Estado de São Paulo. Campinas: Instituto Agronômico, 1997.59 p. (Boletim Técnico, 166)

SOUSA, J. S. I. de, Uvas para o Brasil. 2. ed. Piracicaba: Fealq, 1996. $791 \mathrm{p}$.

TERRA, M. M; PIRES, E. J. P.; COELHO, S. M. B. M.; PASSOS, I. R. S.; SANTOS, R. R.; POMMER, C. V.; SILVA, A. C. P.; RIBEIRO, I. J.A. Porta-enxertos para o cultivar Máximo IAC 138-22 de uvas de vinho em Monte Alegre do Sul, SP. Bragantia, Campinas, v.49, n.2, p.363369, 1990.

TERRA, M. M.; PIRES, E. J. P.; POMMER, C. V.; PASSOS, I. R. S.; MARTINS, F. P.; RIBEIRO, I. J.A. Comportamento de porta-enxertos para o cultivar de uva de mesa Niagara Rosada em Jundiaí-SP. In: CONGRESSO BRASILEIRO DE FRUTICULTURA, 9, 1987, Campinas. Anais... Campinas: Sociedade Brasileira de Fruticultura, 1988. v.2.p.721-725.

TERRA, M. M.; POMMER, C. V.; PIRES, E. J. P.; RIBEIRO, I. J. A.; GALLO, P. B.; PASSOS, I. R. S. Produtividade de cultivares de uvas para suco sobre diferentes porta-enxertos IAC em Mococa-SP. Revista Brasileira de Fruticultura, Jaboticabal, v.23, n.2, p.382386, 2001

TERRA, M. M.; POMMER, C. V.; PIRES, E. J.P.; RIBEIRO, I. J.A.; GALLO, $\mathrm{P}$. B. Production de trois variétés à raisins de table sur quatre portegreffes au Brésil. Bulletin l'O.I.V., v.75, n.851-852, p.4-20, 2002. 